\title{
Extended Inferior Turbinate Flap for Endoscopic Reconstruction of Skull Base Defects
}

\author{
Garret W. Choby ${ }^{1}$ Carlos D. Pinheiro-Neto ${ }^{1}$ John R. de Almeida ${ }^{1}$ Eugenio Cardenas Ruiz-Valdepeñas ${ }^{2}$ \\ Eric W. Wang ${ }^{1}$ Juan C. Fernandez-Miranda ${ }^{2}$ Paul A. Gardner ${ }^{2}$ Carl H. Snyderman ${ }^{1,2}$ \\ ${ }^{1}$ Department of Otolaryngology, University of Pittsburgh School of \\ Medicine, Pittsburgh, Pennsylvania, United States \\ 2 Department of Neurological Surgery, University of Pittsburgh School \\ of Medicine, Pittsburgh, Pennsylvania, United States \\ Address for correspondence Carl H. Snyderman, MD, MBA, University of \\ Pittsburgh School of Medicine, 200 Lothrop Street, EEI 500, Pittsburgh, PA \\ 15213, United States (e-mail: snydermanch@upmc.edu).
}

J Neurol Surg B 2014;75:225-230.

\begin{abstract}
Keywords

- skull base reconstruction

- endoscopic endonasal approach

- inferior turbinate flap

Objective When the use of the nasoseptal flap for endoscopic skull base reconstruction has been precluded, the posterior pedicle inferior turbinate flap is a viable option for small midclival defects. Limitations of the inferior turbinate flap include its small surface area and limited arc of rotation. We describe a novel extended inferior turbinate flap that expands the reconstructive applications of this flap.

Design Cadaveric anatomical study.

Participants Cadaveric specimens.

Main Outcome Measures Flap size, arc of rotation, and reconstructive applications were assessed.

Results The average width of the flap was $5.46 \pm 0.58 \mathrm{~cm}(7.32 \pm 0.59 \mathrm{~cm}$ with septal mucosa). The average length of the flap was $5.01 \pm 0.58 \mathrm{~cm}(5.28 \pm 0.37 \mathrm{~cm}$ with septal mucosa). The average surface area of the flap was $\sim 27.26 \pm 3.65 \mathrm{~cm}^{2}$ $\left(40.53 \pm 6.45 \mathrm{~cm}^{2}\right.$ with septal mucosa). The extended inferior turbinate flap was sufficient to cover clival defects extending between the paraclival internal carotid arteries. The use of the flap in 22 cadavers and 5 clinical patients is described.

Conclusion The extended inferior turbinate flap presents an additional option for reconstruction of skull base defects when the nasoseptal flap is unavailable.
\end{abstract}

\section{Introduction}

As experience with the endoscopic endonasal approach (EEA) for skull base tumors continues to grow, surgical techniques must continue to evolve to solve increasingly complex challenges. EEA is now used for many extensive lesions that were once thought to be resectable only by traditional open approaches. Along with the increased use of EEA for resection, however, comes the need for more versatile techniques for reconstruction.

The challenge for skull base reconstruction involves preventing cerebrospinal fluid (CSF) leaks and exposure of

received

April 13, 2013

accepted after revision

September 9, 2013

published online

April 17, 2014

intracranial contents to intranasal flora by recreating the barrier between the cranial vault and the nasal cavity. ${ }^{1,2}$ Throughout the past 10 years, a variety of vascular and avascular reconstruction techniques have been used. Although small CSF leaks may be repaired with avascular grafting techniques, repair of high-flow leaks has been more successful with vascularized flaps. ${ }^{1,3,4}$

Current options for skull base reconstruction include avascular grafts, intranasal vascular flaps, and novel endoscopic regional flaps. Avascular graft options include fat "bath plug" grafts, free mucosal grafts, and acellular dermal allograft inlays. Intranasal vascular flaps include the nasoseptal

(c) 2014 Georg Thieme Verlag KG Stuttgart · New York
DOI http://dx.doi.org/ 10.1055/s-0033-1358791. ISSN 2193-6331. 
flap (NSF), posterior pedicle inferior turbinate flap (ITF), and middle turbinate flap. Regional vascularized flaps include the pericranial flap, palatal flap, and temporoparietal fascia flap. ${ }^{1,5-8}$

The NSF has served as the primary reconstructive option for anterior skull base defects due to its size and versatility. The NSF is a vascularized pedicled flap based on the posterior septal artery, a branch of the sphenopalatine artery (SPA), which is one of the terminal branches of the internal maxillary artery. ${ }^{3,9}$ The NSF has proven to be a versatile and reliable flap that can readily reconstruct most skull base defects. ${ }^{9,10}$ The NSF has been shown to be successful in limiting postoperative CSF leaks in 94\% of high-flow intraoperative defects. ${ }^{3}$

With the expanded use of EEA for complex and recurrent lesions, however, the NSF may be unavailable as a reconstructive option. Its use may be precluded by prior surgery or tumor involvement of the nasal septum. Other reconstructive options have been described including the ITF. The ITF is a viable option for small midclival defects, but its limitations include its small surface area and limited arc of rotation. Therefore, we have developed a novel extended inferior turbinate flap (EITF) that expands on the applicability of the ITF and may be used when the NSF is unavailable.

\section{Methods}

\section{Anatomical Study}

The anatomical study was completed at the Surgical Neuroanatomy Laboratory of the Department of Neurosurgery at the University of Pittsburgh. The University of Pittsburgh Committee for Oversight of Research Involving the Dead (CORID) granted permission for the work under CORID no. 380. A total of 22 EITFs were dissected. Exclusion criteria were a history of maxillofacial trauma, previous sinonasal surgery, or other sinonasal pathology.

The EITF was designed for harvest in the specimens based on the inferior turbinate (IT) artery, a terminal branch of the SPA. Although a detailed description of the vascular supply to

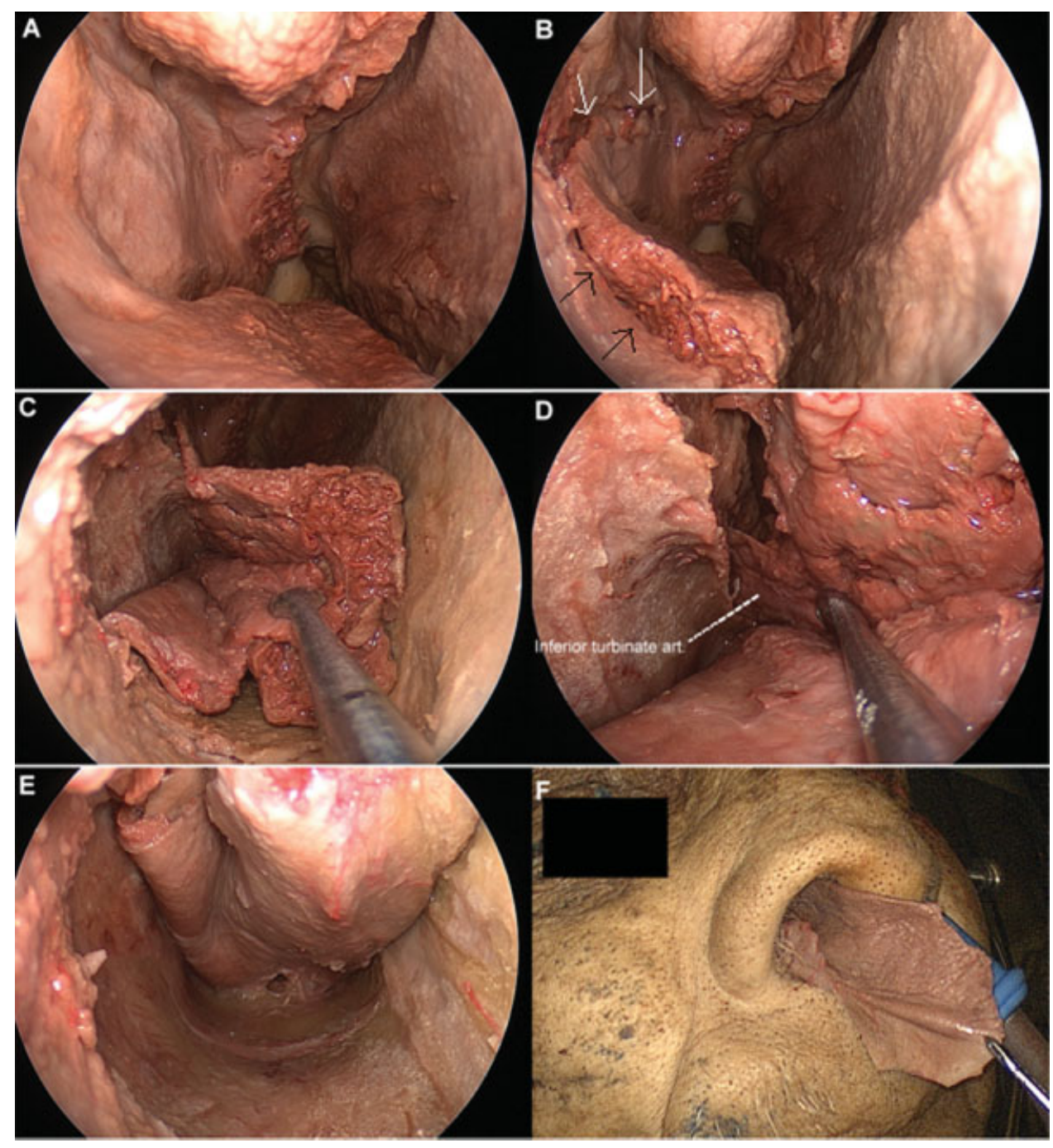

Fig. 1 Endoscopic endonasal dissection and pictures with 0-degree endoscope. Surgical dissection of right-sided extended inferior turbinate flap with addition of the anterior septal mucosa. (A) Resection of the middle turbinate and exposure of the superior surface of the inferior turbinate (IT). (B) Initial incision; white arrows correspond to the initial superior cut; black arrows demonstrate the anterior cut along the head of the IT. (C) Dissection of mucosa from IT. (D) IT artery. (E) Dissection of mucosa from floor of nose and anterior septum. (F) Flap length from nasal vestibule. 
the lateral nasal wall may be found elsewhere, ${ }^{11,12}$ a brief review of pertinent anatomical details is warranted here. The SPA is a terminal branch of the internal maxillary artery. It exits the pterygopalatine fossa via the sphenopalatine foramen into the nasal cavity. It supplies most of the lateral nasal wall including the middle and inferior turbinates via the posterior lateral nasal artery (PLNA), also called the nasolateral artery. The PLNA courses downward and slightly anterior in a remarkably constant position, entering the superior aspect of the lateral attachment of the IT, 1.0 to $1.5 \mathrm{~cm}$ from its posterior margin, where it enters a bony canal and splits into two branches. ${ }^{2,11}$ One branch tends to remain superior and lateral; the other branch traverses anteriorly in an inferomedial position. ${ }^{11}$

\section{Dissection Technique}

Under visualization with a 0-degree endoscope, the nasal cavity was inspected and dissection of the EITF was performed. A maxillary antrostomy was performed and the mucosa covering the ascending process of the palatine bone was elevated. A superior incision was carried from the inferior portion of the maxillary antrostomy anteriorly toward the head of the inferior turbinate. Just anterior to the head of the inferior turbinate, the superior cut was turned 90 degrees inferiorly toward the nasal floor (-Fig. 1A, B) and progressed medially toward the nasal septum. At the point where the superior incision turned 90 degrees, another small releasing incision along the width of the head of the IT was performed toward its inferomedial edge. This cut facilitated the opening of the IT with the reflection of the IT mucosa inferiorly (-Fig. 1C). A posterior incision was performed in the superior and lateral aspect of the arch of the choana, posterior to the tail of the IT. This incision was prolonged inferiorly and crossed the nasal floor over the hard palate toward the posterior edge of the vomer. Finally, the most medial cut of the flap was performed along the junction between the septum and the nasal floor from posterior to anterior ( - Fig. 1D, E, F). Note that the superior limit of the flap corresponded to the inferior limit of the maxillary antrostomy. For the first 17

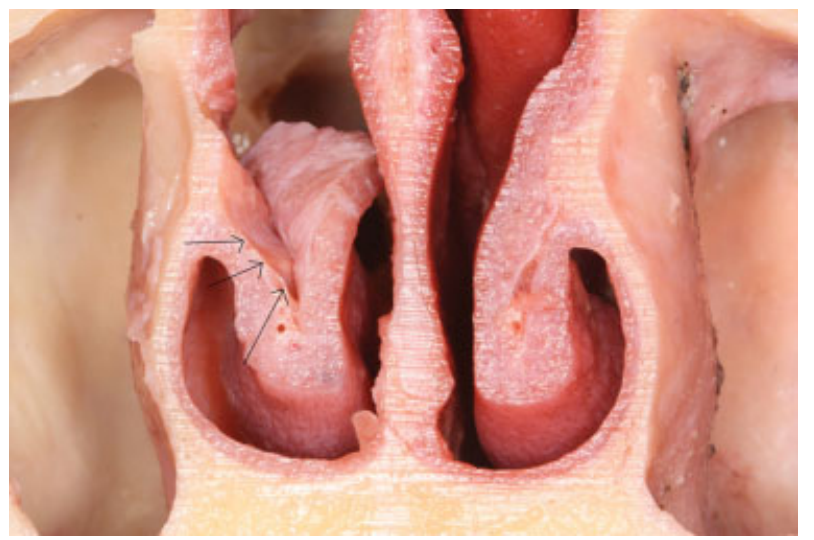

Fig. 2 Cross section of dissection of right inferior turbinate mucosa from the bone (arrows). specimens, the septum was chosen as the limit of this incision to simulate cases where the septal mucosa is not available.

In an additional five specimens, the septal mucosa was included as the most medial portion of the flap. The posterior-superior cut along the septum was performed at the level of the head of the middle turbinate. The septal mucosa covering the quadrangular cartilage was elevated together with the mucosa of the nasal floor. The anterior cut was performed at the level of the caudal edge of the septum, and the most anterior-superior cut was performed at the level of the nasal dorsum. These two incisions are the same used to harvest the nasoseptal flap. Therefore, these flaps included the mucosa of the IT, floor of the nose, and the anterior nasal septum.

After the cuts were completed as just described, the meatal mucosa of the IT was dissected from the IT bone. The mucosa was then opened like a book, and the bone was removed in pieces because of its fragility. Careful dissection is important to avoid a tear at the junction between the meatal mucosa and the medial mucosa of the IT (-Fig. 2). After a complete resection of the IT bone was achieved, a sharp cut was made across the nasolacrimal duct where it opens in the inferior meatus. The mucosa of the lateral wall of the inferior meatus was elevated in continuity with the IT mucosa. At this time, the EITF was elevated from the floor of the nose in a subperiosteal plane in an anterior to posterior manner, toward the pedicle of the flap (-Fig. 1E and -Fig. 3). Care was taken to protect the mucosa that encloses the IT artery (-Fig. 1D) branching from the PLNA and entering its bony canal near the posterior tip of the IT.

After the flap was harvested, a wide bilateral sphenoidotomy was performed with drilling of the sphenoid floor to the clivus and removal of all sphenoid septations. The mucosa of the nasopharynx was resected to expose the lower clivus (-Fig. 4A, B, C). The EITF was rotated toward the clivus to assess the arc of rotation and coverage ( $\mathbf{F i g}$. 4D, E, F). The pedicle of the flap was then transected, and the flap was removed for measurements of the length, width, and surface area (-Fig. 5 and - Fig. 6).

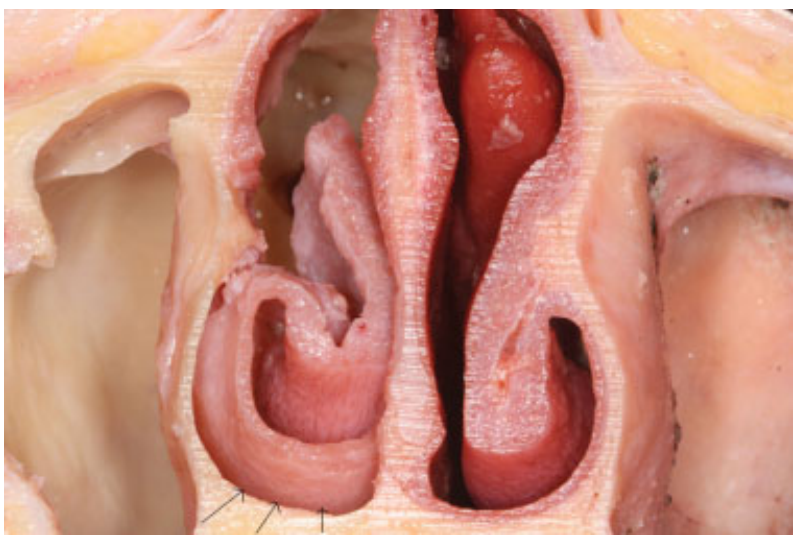

Fig. 3 Cross section of dissection of right floor of nose (arrows). 

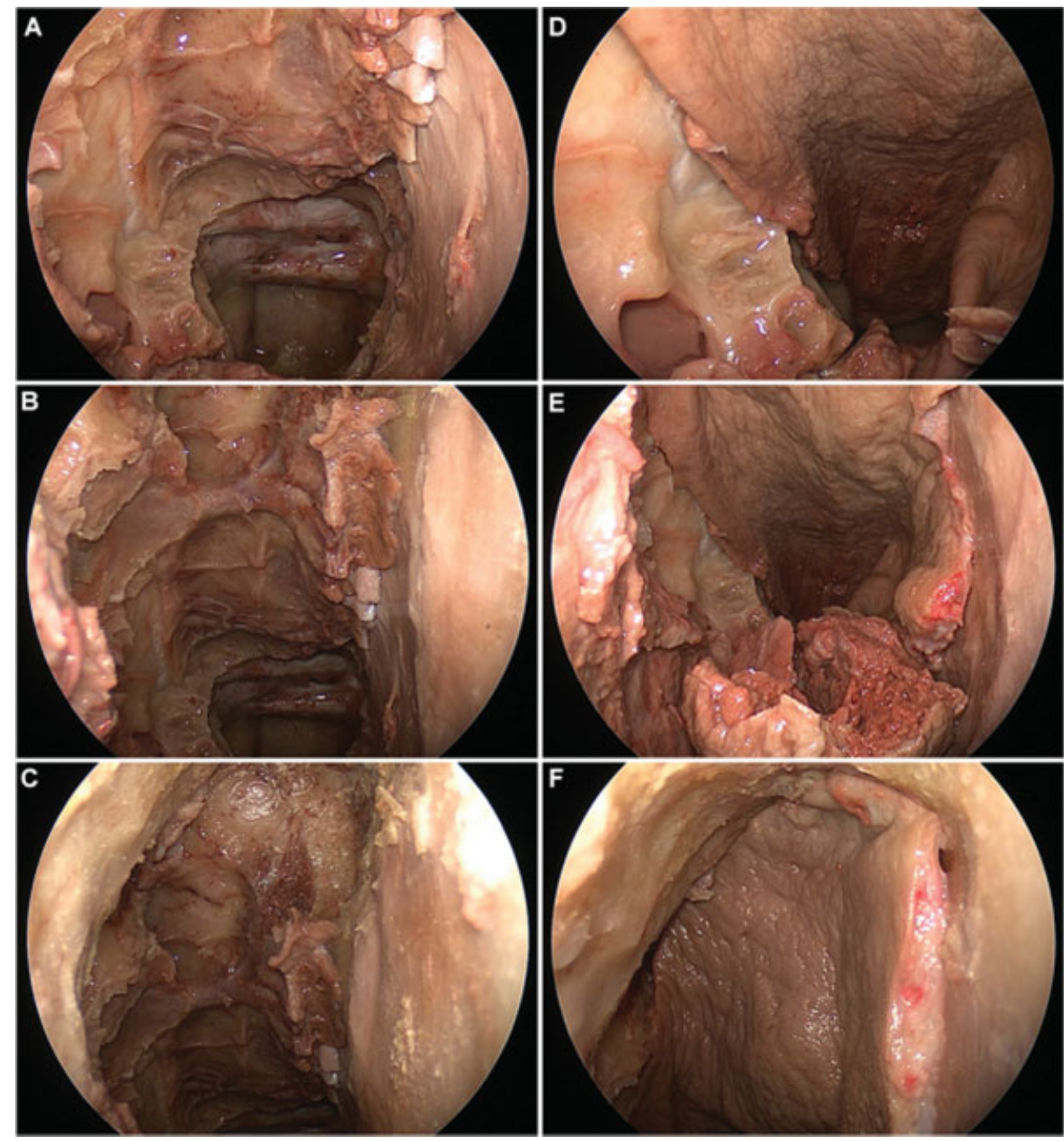

Fig. 4 Endoscopic endonasal dissection and pictures with 0-degree endoscope. (A-C) Complete anterior and posterior ethmoidectomy, large sphenoidotomy, and frontal sinusotomy (Draf IIb) on the right side for exposure of the entire skull base from the sella to the frontal sinus. (D-F) Rotation and placement of extended inferior turbinate flap with the anterior septal mucosa added.

\section{Results}

The average width of the flap, as defined as the widest measurement at the anterior margin of the flap, was $5.46 \pm 0.58 \mathrm{~cm}(7.32 \pm 0.59 \mathrm{~cm}$ with septal mucosa). The

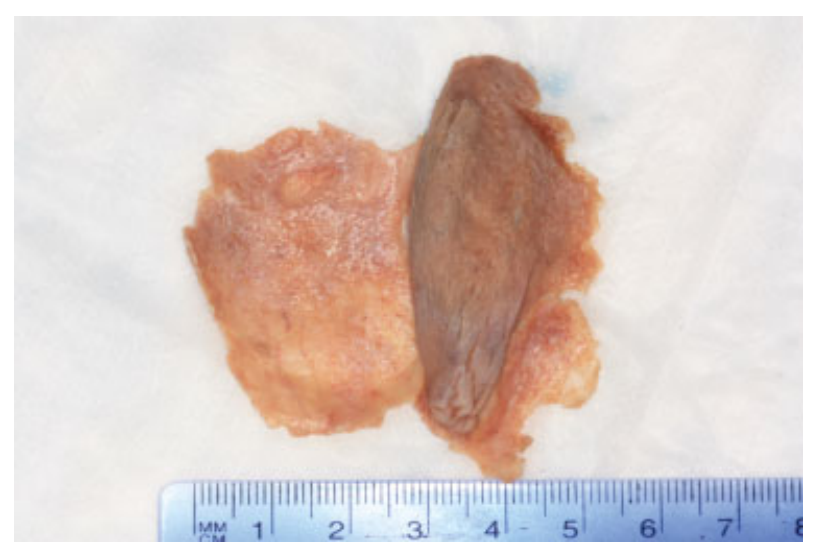

Fig. 5 Extended inferior turbinate flap: right side. average length of the flap, as defined as the longest measurement at the lateral margin of the flap, was $5.01 \pm 0.58 \mathrm{~cm}$ $(5.28 \pm 0.37 \mathrm{~cm}$ with septal mucosa). The average approximate surface area of the flap was $27.26 \pm 3.65 \mathrm{~cm}^{2}$ (40.53 $\pm 6.45 \mathrm{~cm}^{2}$ with septal mucosa). This was defined as

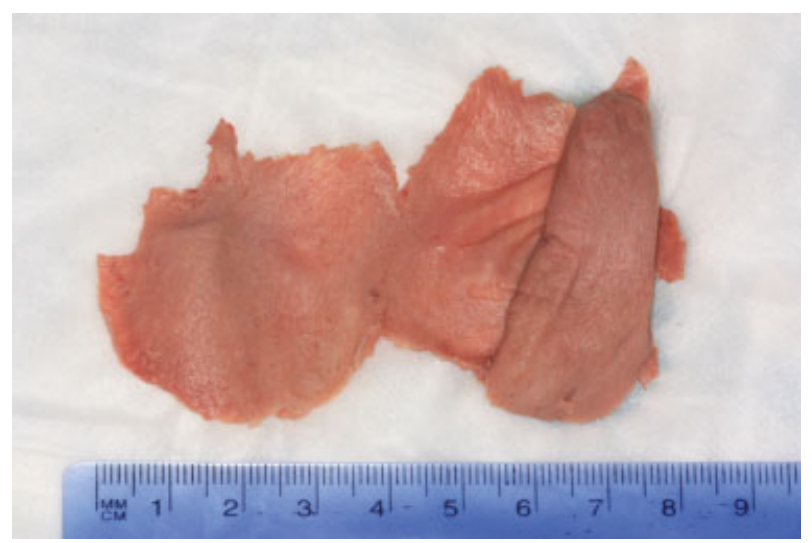

Fig. 6 Extended inferior turbinate flap with septal mucosa: left side. 
Table 1 Clinical use of the extended inferior turbinate flap

\begin{tabular}{|l|l|l|l|l|l|}
\hline Patient no. & Indication & Reason for EITF & Flap type & Resulting defect & Result \\
\hline 1 & Pituitary adenoma & Prior NSF & EITF + septum & Paraclival ICA & No complication \\
\hline 2 & Chordoma & Prior NSF & EITF + septum & Clival defect & No complication \\
\hline 3 & CSF leak & Prior NSF & EITF & Clival defect & No complication \\
\hline 4 & CSF leak & Prior NSF & EITF & Clival defect & No complication \\
\hline 5 & Chondrosarcoma & Prior NSF & EITF & Clival defect & CSF leak \\
\hline
\end{tabular}

Abbreviations: CSF, cerebrospinal fluid; EITF, extended inferior turbinate flap; ICA, internal carotid artery; NSF, nasoseptal flap.

multiplying the widest width measurement by the longest length measurement of each flap, allowing for an approximation of surface area.

For the flaps on the left side, the arc of rotation was clockwise. Likewise, the rotation was counterclockwise for the flaps on the right side. In both situations, the IT mucosa was positioned covering the lower clivus, and the extension of the nasal floor mucosa was used to cover the superior part of the defect. Thus the angle of rotation was more favorable and smaller when compared with the 180-degree angle necessary for the traditional ITF to reach the clivus. The EITF was sufficient to cover the lower and middle clivus between the Eustachian tubes inferiorly and paraclival internal carotid arteries superiorly. The anterior septum mucosa present in five cases improved the coverage superiorly toward the upper clivus.
In addition, the EITF was used in five clinical patients to date as described in - Table $\mathbf{1}$, along with the results.

\section{Discussion}

The ITF was first described by Fortes et al in $2007 .^{2}$ This flap is based on the IT artery, a terminal branch of the SPA. The ITF is a reconstructive option when a NSF is not available due to prior surgery or tumor involvement. The standard ITF is suitable for small midclival defects but is limited due to the small surface area, lack of conformity of the conchal mucosa, and a limited arc of rotation. In an effort to expand the applications of the ITF, we have explored mucosal extensions of the flap in human cadavers.

The size of the ITF has been reported to be $5.4 \mathrm{~cm}$ in length and $2.2 \mathrm{~cm}$ in width. ${ }^{13}$ The length of the EITF is consistent
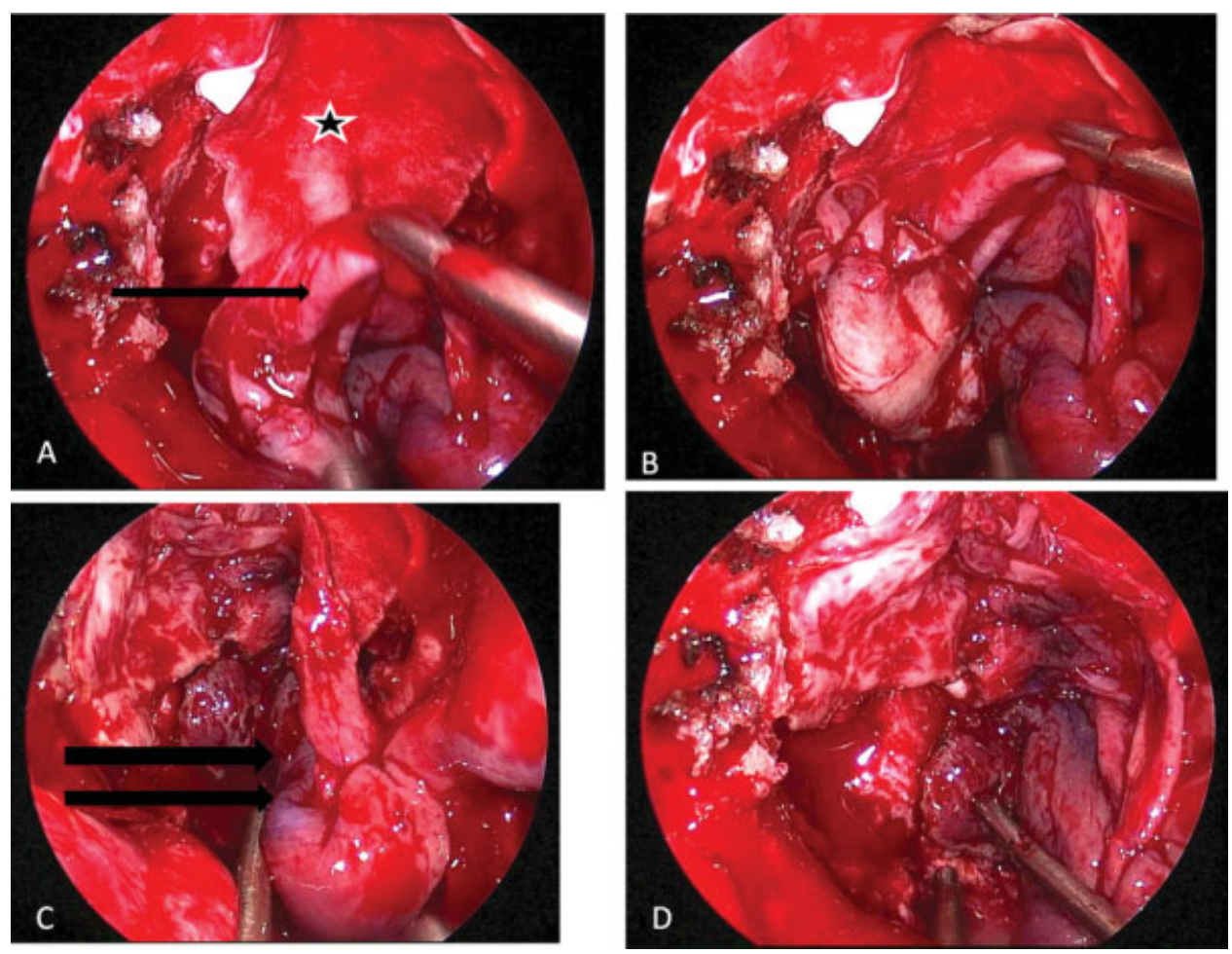

Fig. 7 Intraoperative images of the extended inferior turbinate flap (EITF) in clinical patients. (A) Inset of the EITF (single arrow) with AlloDerm underlay (star). (B) Positioning of the EITF. (C) Inset flap with pedicle at the root of inferior turbinate (double arrows). (D) Final inset. 
with the previously described ITF; the width is $250 \%$ that of the ITF. This difference is accounted for by inclusion of the mucosa of the nasal floor, thus increasing its mean surface area to $27.26 \mathrm{~cm}^{2}$. The surface area was sufficient to cover defects spanning the clivus from one paraclival internal carotid artery to the other. The EITF compares favorably with the NSF for reconstruction of infrasellar defects. The NSF has a mean surface area of $25 \mathrm{~cm}^{2,10,14}$ The EITF is slightly larger.

When the septal mucosa is available for use with the EITF, the mean surface area increases to $40.53 \mathrm{~cm}^{2}$, significantly larger than the mean surface area reported for the NSF. The addition of the nasal mucosa also decreases the arc of rotation by providing a longer flap with greater reach. In addition to infrasellar defects, the larger flap can provide effective coverage of defects of the anterior cranial base (-Fig. 4).

Although the injected specimens demonstrate that the PLNA branch of the sphenopalatine artery supplies the mucosa of the inferior turbinate and inferior meatus, it is unclear how well it supplies the mucosa of the EITF. ${ }^{11,12}$ It is likely that most of the septal mucosa has a random blood supply and would benefit from a wide pedicle that includes the entire nasal floor mucosa. Although the distal part of the flap may be random, this does not preclude its use as an effective vascularized reconstructive flap. Thus the mucosa of the IT functions as a vascularized pedicled flap with an extension (nasal floor and septal mucosa) that likely benefits from a random blood supply.

The dissection of the EITF can be technically challenging, especially raising the tightly adherent mucosa from the IT bone. However, we did not encounter significant problems with perforation of the flaps likely due to the thick nature of the IT mucosa, in addition to our method of opening the IT like a book and removing the bone piecemeal as we described in the technique section.

In patients who have had a prior NSF, the vascular pedicle for the ITF is usually preserved on the side of the prior flap. If a revision surgery is performed and a new reconstruction is necessary, the EITF provides adequate coverage for most infrasellar defects. The EITF could also be combined with a NSF from the same or alternate sides to provide two separate reconstructive flaps. Additional experience is needed before passing judgment on its clinical effectiveness.

In our experience using the EITF in five clinical patients, it has been successful in four cases in preventing a postoperative CSF leak. Each of these cases was a revision case, and the ETIF was selected for use due to a previously utilized NSF. In all cases, the EITF proved to be a large and versatile flap that was adequate to cover the defect (-Fig. 7). Postoperatively, we have not noted an increased amount of crusting when compared with standard NSF patients. When MT mucosa is available, it is used as a mucosal free graft to the anterior septum, which may help expedite the healing process.

\section{Conclusion}

The EITF provides an additional reconstructive option when the NSF is unavailable. It provides the benefits of a local pedicled vascularized mucosal flap while improving on the shortcomings of the ITF. Its large surface area and improved arc of rotation make it a versatile flap that can cover infrasellar defects in the clival region and provide coverage of the paraclival internal carotid arteries.

\section{Disclaimer}

No financial acknowledgments are pertinent to this study.

\section{Note}

This article was presented at the Academy of Otolaryngology-Head and Neck Surgery Annual Meeting; September 10, 2012; Washington, DC.

\section{References}

1 Patel MR, Shah RN, Snyderman $\mathrm{CH}$, et al. Pericranial flap for endoscopic anterior skull-base reconstruction: clinical outcomes and radioanatomic analysis of preoperative planning. Neurosurgery 2010;66(3):506-512; discussion 512

2 Fortes FS, Carrau RL, Snyderman CH, et al. The posterior pedicle inferior turbinate flap: a new vascularized flap for skull base reconstruction. Laryngoscope 2007;117(8):1329-1332

3 Zanation AM, Carrau RL, Snyderman CH, et al. Nasoseptal flap reconstruction of high flow intraoperative cerebral spinal fluid leaks during endoscopic skull base surgery. Am J Rhinol Allergy 2009;23(5):518-521

4 Carrau RL, Snyderman CH, Kassam AB. The management of cerebrospinal fluid leaks in patients at risk for high-pressure hydrocephalus. Laryngoscope 2005;115(2):205-212

5 Hadad G, Bassagasteguy L, Carrau RL, et al. A novel reconstructive technique after endoscopic expanded endonasal approaches: vascular pedicle nasoseptal flap. Laryngoscope 2006;116(10): $1882-1886$

6 Kassam A, Carrau RL, Snyderman CH, Gardner P, Mintz A. Evolution of reconstructive techniques following endoscopic expanded endonasal approaches. Neurosurg Focus 2005;19(1):E8

7 Neligan PC, Mulholland S, Irish J, et al. Flap selection in cranial base reconstruction. Plast Reconstr Surg 1996;98(7):1159-1166; discussion 1167-1168

8 Snyderman CH, Janecka IP, Sekhar LN, Sen CN, Eibling DE. Anterior cranial base reconstruction: role of galeal and pericranial flaps. Laryngoscope 1990;100(6):607-614

9 Pinheiro-Neto CD, Ramos HF, Peris-Celda M, et al. Study of the nasoseptal flap for endoscopic anterior cranial base reconstruction. Laryngoscope 2011;121(12):2514-2520

10 Pinheiro-Neto CD, Prevedello DM, Carrau RL, et al. Improving the design of the pedicled nasoseptal flap for skull base reconstruction: a radioanatomic study. Laryngoscope 2007;117(9): 1560-1569

11 Padgham N, Vaughan-Jones R. Cadaver studies of the anatomy of arterial supply to the inferior turbinates. J R Soc Med 1991;84(12): 728-730

12 Hadar T, Ophir D, Yaniv E, Berger G. Inferior turbinate arterial supply: histologic analysis and clinical implications. J Otolaryngol 2005;34(1):46-50

13 Harvey RJ, Sheahan PO, Schlosser RJ. Inferior turbinate pedicle flap for endoscopic skull base defect repair. Am J Rhinol Allergy 2009; 23(5):522-526

14 Pinheiro-Neto CD, Pinheiro SD. Use of pedicle flaps for skull base reconstruction after expanded endonasal approaches. Int Arch Otorhinolaryngol 2007;11(3):324-329 